\title{
Navier slip and suction/injection effects on oscillatory flow of nanofluid with fluid-particle interaction in a channel
}

\author{
Jafar Hasnain ${ }^{1,}$, Mariam Sheikh², and Zaheer Abbas $^{3}$ \\ ${ }^{1}$ Department of Computer Sciences, Bahria University Islamabad Campus, Islamabad 44000, Pakistan \\ ${ }^{2}$ Department of Mathematics, University of Sialkot, Sialkot 51310, Pakistan \\ ${ }^{3}$ Department of Mathematics, The Islamia University of Bahawalpur, Bahawalpur 63100, Pakistan
}

Keywords: Nanofluid, dust particles, oscillatory flow, suction/injection, heat absorption/generation.

Topic: Heat and Mass Transfer in Particle-Laden flows

\begin{abstract}
In this paper, the slip and porosity effects on the channel walls are studied for the oscillatory flow of heat-absorbing/generating dusty nanofluid flowing through a porous medium. The channel is vertical and exposed to the transverse magnetic field and thermal radiation. The base fluid is water with silver as nanoparticles. The basic equations of the flow problem, which appeared to be dimensional, are remodelled in the dimension-less form with the help of non-dimensional variables. The obtained equations are solved analytically using the variable separable method. The graphs are presented to show the impact of these parameters on the flow fields, skin friction, heat transmission rate and discussed in detail. Results reveal that the flow velocities of fluid and particles for suction are greater than in the case of injection. The fluid velocity upsurges with the improved values of the velocity slip parameter.
\end{abstract}

\section{Nomenclature}

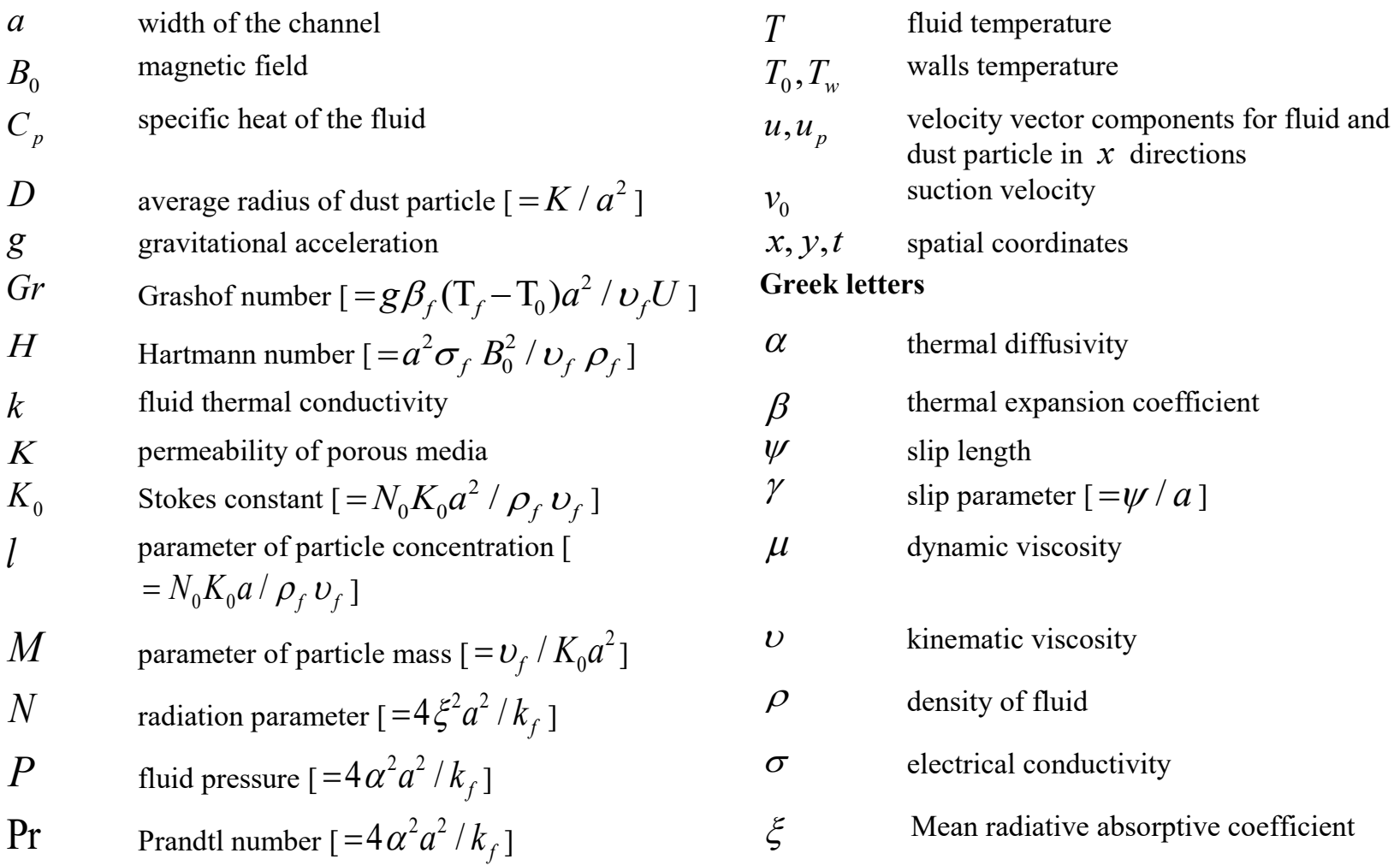

\footnotetext{
${ }^{*}$ Corresponding author: Tel.: +92 519263268.

e-mail address: jafar_hasnain14@yahoo.com (J. Hasnain)
} 


$\begin{array}{ll}\bar{P} & \begin{array}{l}\text { dimensionless pressure }\left[=a P / v_{f} \rho_{f} U\right] \\ q\end{array} \\ Q^{*} & \text { heat flux of radiation } \\ Q & \text { heat source parameter } \\ & \left.=Q^{*} a^{2} / k_{f}\right] \\ \operatorname{Re} & \text { Reynolds number }\left[=U a / v_{f}\right] \\ S & \text { porous medium shape factor number } \\ S & \text { suction parameter }\left[=-v_{0} a / v_{f}\right]\end{array}$

MHD magnetohydrodynamics

NFs nanofluids

\section{Introduction}

The inherent complexity of fluids in nature as well as in industry has encouraged researchers to introduce unique and innovative fundamental associations to analyze the thermophysical behavior of fluids. In the production of electronic devices with limited thermal conductivity in convection fluids such as ethylene glycol, water and gasoline, there have been a number of problems. Several researchers have focused in recent decades on incorporating nano or micrometre-sized objects in the base liquids to solve this limitation and improve the thermal conductivity. Nanofluid, which aims to enhance thermal conductivity, is formed by combining nanometer-sized particles into the base fluid. While the combination of milli or micrometre-sized particles (dust particles) in the base liquids creates dusty fluid, it, therefore, appears to boost the thermal conductivity of the base liquid. As compared to dusty fluids and nanofluids, Krishnamurthy et al. [1] reported that dusty nanofluids appear to have significant thermal conductivity while examining heat transfer characteristics of dusty fluid condensed with nano-particles past an exponential stretching layer. Affected by the magnetic field, Naramgari and Sulochana [2] studied the flow of nanofluid concentrated with dust particles. They reported increased magnetic field, porosity and dust particle mass concentration parameters contribute to a substantial decrease in the friction factor. In the existence of heat and magnetic field, a numerical result of dusty nanofluid flow was found by Sulochana and Sundeep [3] along with a stretching/shrinking cylinder. They noticed that the rise in dust particles' volume fraction deteriorates the heat transfer rate. Prasannakumara et al. [4] discussed the flow, including dust and nanoparticles with porous media over an extending sheet with heat. They concluded that there is a substantial cooling efficiency of dusty fluid containing copper nanoparticles. Gireesha et al. [5] considered Hall's effects, including magnetic field and heat generation/absorption on two-phase dusty-nanofluid with the observation that on the thermal distribution, Hall and magnetic parameters have opposite effects. Raizah [6] analysed hybrid nanofluid having dust particles moving through an enclosure containing two heated fins. He claimed that at the centre of the top sheet there are the lowest values for the local Nusselt number. Kaneez et al. [7] reported the numerical result of the flow of dust- concentrated micropolar fluid having hybrid nano-particles and presented the result that the parameter of the dust fleck association performs a crucial part in the fluid temperature field. Radhika et al. [8] formulated the mathematical model for the flow of dusty hybrid-nano fluid over a melting plate with the perception that thermal stability of fluid along dust phase improved by raising the concentration of dust particles.

In estimating fluid flows in several industrial and medical systems, the boundary conditions related to the fluid field are quite significant. In polymer melting that often undergoes microscopic wall slippage, the dearth of the noslip condition is very clear. Slip condition performs a crucial role in cleaning interval cavities and artificial heart valves, shear skin, hysteresis impact and spurt. Hayat et al. [9] highlighted the influence of MHD and slip in a channel on nanofluid flow and obtained an analytical solution. Kamel et al. [10] worked on the two-phase stream of dusty liquid in a planar channel with slip boundary condition. The findings suggested that the condition of slip has a major impact under certain concentration ranges. Guria [11] applied the periodic suction on the vertical channel with radiation and slip boundary condition and found that the slip effect has been noticed to minimize the velocity. Panaseti and Georgiou [12] discussed viscoplastic fluid flow in a channel in the channel in slip regime with the observation that there has a huge spike in the mild slip region. The slip parameter reduces the radial velocity. This was reported by Kashyap et al. [13] while studying the flow of UCM fluid past a channel having a chemical reaction and velocity slip boundary condition. Saleem et al. [14] investigated the peristaltic flow of MHD 
Jeffery fluid over a tapered channel with velocity second slip and concluded that a rise in slip parameters decreases the size of the trapping bolus.

In a series of diverse conditions, the heat generation/absorption examination is considered. In these cases, fluids undergo exothermic or endothermic chemical processes. Heat generation/absorption procedures occur when heat energy is collected from the waste of nuclear fuel, or water is heated in a solar collector. It also has applications in packed-bed reactors, radiative cooling of molten steel, semi-conductor wafers, recycling of food products and underground disposal of radioactive waste, electronic chips. The generation or absorption of heat determines both the fluid flow and the heat transfer rate in the moving fluids. Malik et al. [15] discussed the impact of thermal conductivity and heat generation/absorption on Williamson fluid flow over a stretching cylinder. They concluded that the heat source increases the temperature profile, whereas the heat sink shows the opposite behaviour. Pandey and Kumar [16] reported that the temperature profiles are expanded according to each heat generation/absorption parameter value while analysing MHD nanofluid flow through convergent/divergent channels under heat generation /absorption. With heat generation/absorption, Jha et al. [17] investigated fluid flow through the rotating channel. They found out that fluid's temperature decreases with the rise in heat absorption while increases with heat generation. Mishra et al. [18] unveiled the effects of heat generation/absorption through stretching/shrinking channels on MHD nanofluid flow. The highest heat generation/absorption parameter values in the shrinking convergent channel caused a spike in velocity and thermal profiles. Prakesh et al. [19] performed an analysis on the MHD flow of viscous fluid within the vertical channel along with heat generation/absorption and variable properties. They reported that heat transmission rate drops in the left plate with the incorporation of heat generation/absorption and increases in the right plate.

More than one phase stream analysis, in which solid particles are scattered in a base liquid is of enormous interest because of their usefulness. Examples of such flows include solid-fuel rocket nozzles, crude oil cleansing, nuclear reactor cooling, hydro cyclones, electronic devices, cryopreservation and cancer therapies. Suction/injection of the fluid across boundaries is very essential in several fields and practical considerations like aerodynamics, space sciences, film cooling, and control of boundary layer. Taking these applications into account, the purpose of this analysis is to assess the impact of the oscillatory flow of dusty-nano fluid in a channel. Porous media, heat generation/absorption and the slip boundary condition are also considered.

\section{Problem development}

The laminar flow is considered under the impact of radiative heat flux in a permeable media, saturated with incompressible dusty fluid. The medium is drawn along the $x$-axis and $y$ axis is kept perpendicular to walls (Fig. 1). The magnetic field strength is parallel to the $y$-axis. The fluid temperature on both walls is different. $T_{f}$ is the initial fluid temperature. $T_{0}$ is the left wall temperature whereas $T_{w}$ is the temperature at the right wall. $R e$ is very small and hence the induced magnetic field becomes insignificant. The fluid is drawn from the left wall and injected from the right wall. With these assumptions the flow equations are

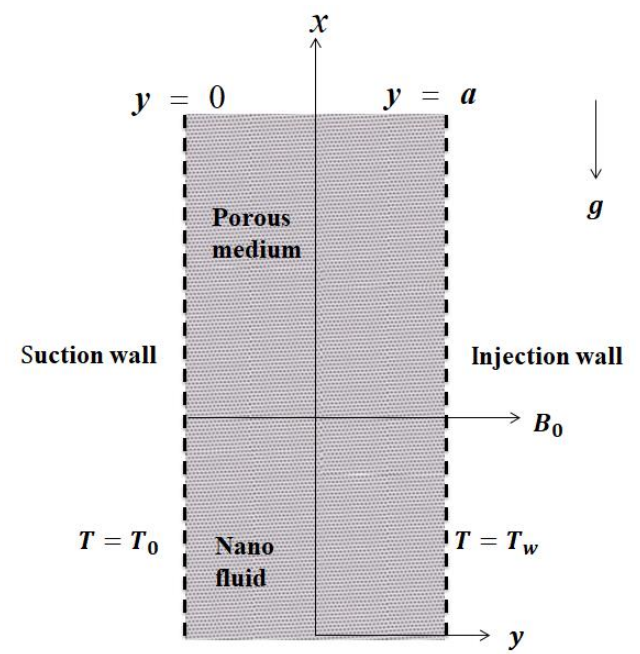

Fig.1: Geometry of the channel.

$u_{t}-v_{0} u_{y}=-\frac{1}{\rho_{n f}} P_{x}+v_{n f} u_{y y}-\frac{v_{n f}}{K} u+$

$\frac{N_{0} K_{0}}{\rho_{n f}}\left(u_{p}-u\right)-\frac{\sigma_{n f}}{\rho_{n f}} B_{0}^{2} u+g \beta\left(T-T_{0}\right)$,

$\left(u_{p}\right)_{t}=K_{0}\left(u_{p}-u\right)$,

$T_{t}-v_{o} T_{y}=\frac{k_{n f}}{\left(\rho c_{p}\right)_{n f}} T_{y y}-\frac{1}{\left(\rho c_{p}\right)_{n f}} q_{y}+\frac{Q^{*}}{\left(\rho c_{p}\right)_{n f}}\left(T-T_{0}\right)$,

with initial and surface constraints

$$
\begin{gathered}
u=u_{p}=0, \quad T=T_{f} \quad \text { at } \quad(y, t)=(y, 0), \\
u=u_{p}=0, \quad T=T_{w} \quad \text { at } \quad(y, t)=(a, t), \\
u=\psi u_{y}, \quad u_{p}=0, \quad T=T_{0} \quad \text { at } \quad(y, t)=(0, t),
\end{gathered}
$$

where $T_{w}=T_{0}+\left(T_{f}-T_{0}\right) e^{i \omega t}$.

Assuming fluid to be thin optically and having low density relatively, the expression for RHF is given as [20]

$$
q_{y}=4 \xi^{2}\left(T_{0}-T\right) \text {. }
$$

The forthcoming non-dimensional quantities are used. 
$\bar{x}=\frac{x}{a}, \quad \bar{y}=\frac{y}{a}, \quad \bar{u}=\frac{u}{U}, \quad \theta=\frac{T-T_{0}}{T_{f}-T_{0}}, \quad \bar{t}=\frac{t U}{a}$,

$D a=\frac{K}{a^{2}}, M=\frac{v_{f}}{K_{0} a^{2}}, l=\frac{N_{0} K_{0} a^{2}}{\rho_{f} v_{f}}, \quad \operatorname{Re}=\frac{U a}{v_{f}}$,

$\operatorname{Pr}=\frac{v_{f} \rho_{f} c_{p}}{k_{f}}, \quad N^{2}=\frac{4 \alpha^{2} a^{2}}{k_{f}}, \bar{u}_{p}=\frac{u_{p}}{U}, s^{2}=\frac{1}{D a}$, (6)

$Q=\frac{Q^{*} a^{2}}{k_{f}}, S=\frac{-v_{0} a}{v_{f}}, \mathrm{Gr}=\frac{g \beta_{f}\left(\mathrm{~T}_{f}-\mathrm{T}_{0}\right) a^{2}}{v_{f} U}$,

$\overline{\mathrm{P}}=\frac{a P}{v_{f} \rho_{f} U}, \quad H^{2}=\frac{a^{2} \sigma_{f} B_{0}^{2}}{v_{f} \rho_{f}}, \quad \gamma=\frac{\psi}{a}$.

The expressions for $\mu_{n f}, \rho_{n f}, \alpha_{n f}, \beta_{n f}, \sigma_{n f},\left(\rho c_{p}\right)_{n f}$ and $k_{n f}$ are expressed as [21]

$\alpha_{n f}=\frac{k_{n f}}{\left(\rho C_{p}\right)_{n f}}, \quad \rho_{n f}=(1-\phi) \rho_{f}+\phi \rho_{s}, \quad \mu_{n f}=\frac{\mu_{f}}{(1-\phi)^{2.5}}$,

$\frac{k_{n f}}{k_{f}}=\frac{k_{s}+2 k_{f}-2 \phi\left(k_{f}-k_{s}\right)}{k_{s}+2 k_{f}+\phi\left(k_{f}-k_{s}\right)}, \quad\left(\rho C_{p}\right)_{n f}=(1-\phi)\left(\rho C_{p}\right)_{s}$,

$\beta_{n f}=(1-\phi) \beta_{f}+\phi \beta_{s}, \frac{\sigma_{n f}}{\sigma_{f}}=1+\frac{3 \phi\left(\frac{\sigma_{s}}{\sigma_{f}}-1\right)}{\left(\frac{\sigma_{s}}{\sigma_{f}}+2\right)-\phi\left(\frac{\sigma_{s}}{\sigma_{f}}-1\right)}$.

Table 1: Thermo-physical properties of NFs and NPs.

\begin{tabular}{|c|c|c|c|c|c|}
\hline $\begin{array}{c}\text { Liquids and } \\
\text { nano } \\
\text { particles }\end{array}$ & $\begin{array}{l}\rho \\
\left(K g / m^{3}\right)\end{array}$ & $\begin{array}{l}c_{p} \\
(k / k g)\end{array}$ & $\begin{array}{l}k \\
(w K / m)\end{array}$ & $\begin{array}{l}\beta^{*} 10^{-5} \\
\left(K^{-1}\right)\end{array}$ & $\begin{array}{l}\sigma \\
(S / m)\end{array}$ \\
\hline Pure Water & 997.1 & 4179 & 0.613 & 21 & $5.5^{*} 10^{-6}$ \\
\hline Silver (Ag) & 10500 & 235.5 & 429 & 1.89 & $63 * 10^{7}$ \\
\hline
\end{tabular}

The flow equations along with the surface constraints in nondimensional form can be written as

$$
\begin{aligned}
& \operatorname{Re} u_{t}=-\frac{1}{Q_{1}} P_{x}+S u_{y}+\frac{1}{Q_{1} Q_{2}} u_{y y}- \\
& \left(\frac{H^{2} Q_{4}}{Q_{1}}+\frac{s^{2}}{Q_{1} Q_{2}}+\frac{l}{Q_{1}}\right) u+\frac{l}{Q_{1}} u_{p}+G r Q_{3} \theta,
\end{aligned}
$$

$$
\operatorname{Re} M\left(u_{p}\right)_{t}=u-u_{p} \text {, }
$$

$\operatorname{Re} \operatorname{Pr} Q_{5} \theta_{t}-S \operatorname{Pr} Q_{5} \theta_{y}=Q_{6} \theta_{y y}+N^{2} \theta+Q \theta$,

with surface constraints as

$$
\begin{gathered}
u=u_{p}=0, \quad \theta=1 \quad \text { at } \quad(y, t)=(y, 0), \\
u=u_{p}=0, \quad \theta=e^{i \omega t} \quad \text { at } \quad(y, t)=(1, t), \\
u=\gamma u_{y}, \quad u_{p}=0, \quad \theta=0 \quad \text { at } \quad(y, t)=(0, t) .
\end{gathered}
$$

The bars are discarded for simplicity.

\section{Method of Solution}

The solution of the Eqs. (8)-(10) is obtained for pure oscillatory flow by letting

$$
\begin{gathered}
-P_{x}=\lambda e^{i \omega t}, \quad u(y, t)=u_{0}(y) e^{i \omega t}, \\
u_{p}(y, t)=u_{p 0}(y) e^{i \omega t}, \quad \theta(y, t)=\theta_{0}(y) e^{i \omega t} .
\end{gathered}
$$

Substituting the values from equation (12) into equations (8)(11) we obtain

$$
\begin{aligned}
& \frac{1}{Q_{7}}\left(u_{0}\right)_{y y}+S\left(u_{0}\right)_{y}-Q_{8}^{2} u_{0}=-\frac{\lambda}{Q_{1}}-G r \theta_{0} Q_{3}, \\
& \qquad u_{p 0}=\frac{u_{0}}{(1+i \omega \operatorname{Re} M)}, \\
& Q_{6}\left(\theta_{0}\right)_{y y}+\operatorname{Pr} S Q_{5}\left(\theta_{0}\right)_{y}+Q_{9}^{2} \theta_{0}=0, \\
& \text { with } \\
& \qquad u_{0}=u_{p_{0}}=0, \quad \theta_{0}=1 \text { at } \quad(y, t)=(y, 0), \\
& u_{0}=u_{p_{0}}=0, \quad \theta_{0}=e^{i \omega t} \quad \text { at } \quad(y, t)=(1, t), \\
& u_{0}=\gamma u_{0 y}, \quad u_{p_{0}}=0, \quad \theta_{0}=0 \text { at }(y, t)=(0, t) .
\end{aligned}
$$

On solving equation (15) with constraints (16), the expression for fluid temperature is obtained as

$$
\theta(y, t)=e^{i \omega t}\left(C_{1} e^{Q_{11} y}+C_{2} e^{Q_{12} y}\right),
$$

The expressions for fluid and dusty particles velocities are obtained after solving Eqs. (13) and (14) respectively along with the constraints (16) and given as

$$
\begin{aligned}
& u(y, t)=e^{i \omega t}\left(\begin{array}{l}
C_{3} e^{Q_{13} y}+C_{4} e^{Q_{14} y}+ \\
Q_{15}+Q_{16} e^{Q_{11} y}+Q_{17} e^{Q_{12} y}
\end{array}\right), \\
& u_{p}(y, t)=\frac{1}{1+i \omega \operatorname{Re} M} e^{i \omega t}\left(\begin{array}{l}
C_{3} e^{Q_{13} y}+C_{4} e^{Q_{14} y}+ \\
Q_{15}+Q_{16} e^{Q_{11} y}+Q_{17} e^{Q_{12} y}
\end{array}\right) .
\end{aligned}
$$

The quantities of interest for engineering point of view are surface friction $C_{f}$ and Nusselt number $N u$, which are expressed as

$$
C_{f}=\frac{a \tau_{f}}{\rho v U}=\left.u_{y}\right|_{y=0,1}=e^{i \omega t}\left(\begin{array}{l}
C_{3} Q_{13} e^{Q_{13} y}+C_{4} Q_{14} e^{Q_{14} y} \\
+Q_{16} Q_{11} e^{Q_{11} y}+Q_{17} Q_{12} e^{Q_{12} y}
\end{array}\right),
$$


where $\tau_{f}=\rho v u_{y}$ at $y=a=1$ and $y=0$ represent the shear stresses of fluid at the right and left walls of the channel respectively, and

$$
N u=\frac{a q_{w}}{k\left(T_{f}-T_{0}\right)}=-\left.\theta_{y}\right|_{y=0,1}=-e^{i \omega t}\left(\begin{array}{l}
C_{1} Q_{11} e^{Q_{11} y} \\
+C_{2} Q_{12} e^{Q_{12} y}
\end{array}\right),
$$

where $q_{w}=-k T_{y}$ at $y=a=1$ and $y=0$ represent the heat fluxes at the right and left walls of the channel respectively. The Appendix contains the constants that appear in the above expressions.

\section{Results and discussion}

The expressions for the velocity of dust particles as well as velocity and temperature of fluid are obtained by solving flow equations with the surface constraints analytically using the method of separating variables. Graphs are generated to demonstrate the influence of the various parameters on the velocity along with temperature profiles.

Figure 2 is designed to present the behavior of fluid velocity $u$ and particle velocity $u_{p}$ with the variation in $S$.

The Fig. shows that both $u$ and $u_{p}$ is increasing with an increase in suction parameter $S(>0)$ however $u$ decreases with a raise in injection parameter $S(<0)$. Fig. 3 displays the variation in $u$ and $u_{p}$ with $\gamma$ for two values of $S$. It can be seen that both velocities $u$ and $u_{p}$ increases with the increment in $\gamma$ for both cases of suction and injection. However, the flow field for $S(>0)$ is higher relative to the flow field for $S(<0)$. It is also observed that the variation in $u$ takes place near the left wall $(y=0)$ as the velocity slip is considered only at this wall. Fig. 4 presents the changing behavior of $u$ and $u_{p}$ with $\phi$ for both cases of suction and injection. It is clear from this Fig. that an increase in $\phi$ accelerates $u$ and $u_{p}$. The flow field is greater when suction is happening. The graphs show that the flow field is parabolic in nature and the axis of the parabola shifts towards the right wall $(y=1)$ for the case of injection. Fig. 5 presents the change in $u$ and $u_{p}$ with the change in $Q$. The Fig. shows a rise in $u$ and $u_{p}$ when fluid is heat generating $(Q>0)$ while a decrease is noticed when the fluid is heat absorbing $(Q<0)$.

Fig. 6 shows the effects of $\phi, Q$ and $S$ on fluid temperature $\theta$. Fig. 6(a) presents the decreasing behaviour of fluid temperature with an increment in $\phi$ for both heat absorbing and heat-generating fluids. However, the temperature field for heat-generating fluid is greater than the heat adsorbing fluid. Fig. 6(b) is plotted to present the change in $\theta$ with the variation in $Q$. A rise in $\theta$ is noticed with an increase in $(Q>0)$ while $\theta$ reduces with $(Q<0)$. The suction and injection effects on the $\theta$ are plotted in Fig. 6(c). Fig. clearly depicts that suction boosts $\theta$ while it reduces with injection.

Fig. 7 is graphed to present the variation in $C_{f}$ at both walls versus $S(>0)$ for both heat absorbing and generating fluids. Surface friction at the left and right walls are presented in Fig. 7(a) and 7(b) respectively. It can be observed that an increase in $\phi$ raises the magnitude of surface friction at both walls of the channel. $C_{f}$ also enhances with suction for both heat-absorbing and generating liquids. Surface friction at both walls versus $S(>0)$ with the rising values of $\gamma$ are plotted in Fig. 8. An increase in slip reduces the friction at the left wall while it enhances the magnitude at the right wall for both kinds of liquids. Heat transmission rates versus $S(>0)$ at both walls for various values of $\phi$ are presented in Fig. 9. An increase in $\phi$ boosts the heat transmission rate while $S$ declines the heat transfer rate for both heat-absorbing and generating liquids (Fig. 9(a)). Fig. 9(b) shows that the transmission rate is higher for heat-absorbing liquid. Heat transmission rate at the right wall shows an improvement with $S(>0)$ while is reducing with $\phi$. The transmission rate is higher for heat-generating liquid in this case.

(a)

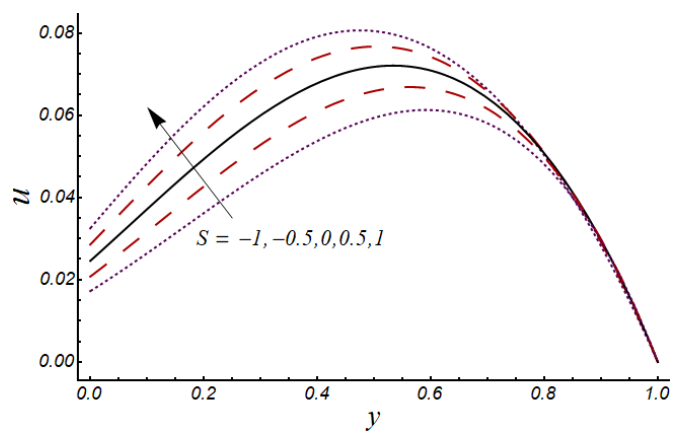

(b) 


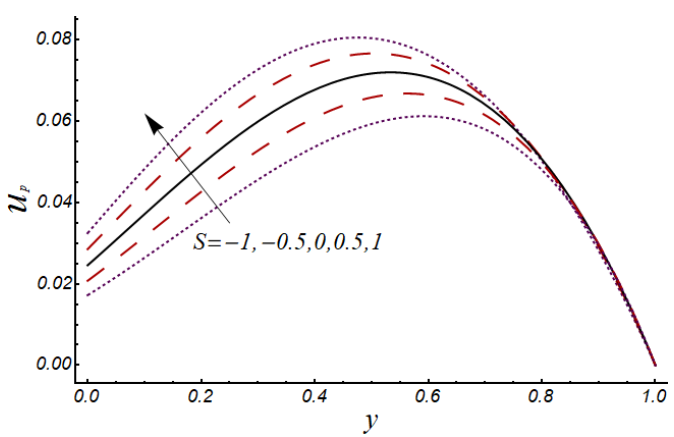

Fig. 2: (a) $u(y, t)$ (b) $u_{p}(y, t)$ for $S$.

(a)

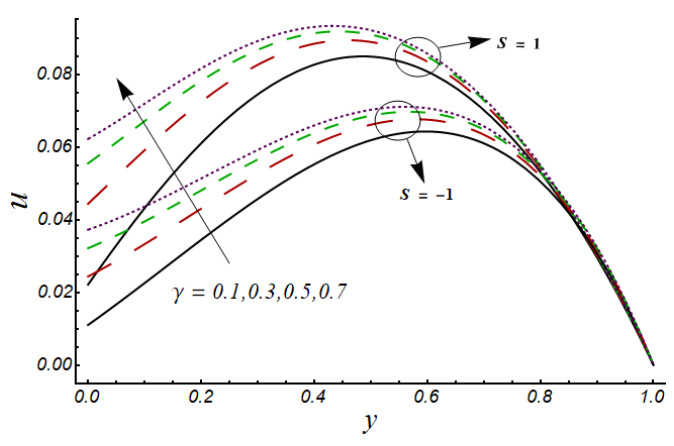

(b)

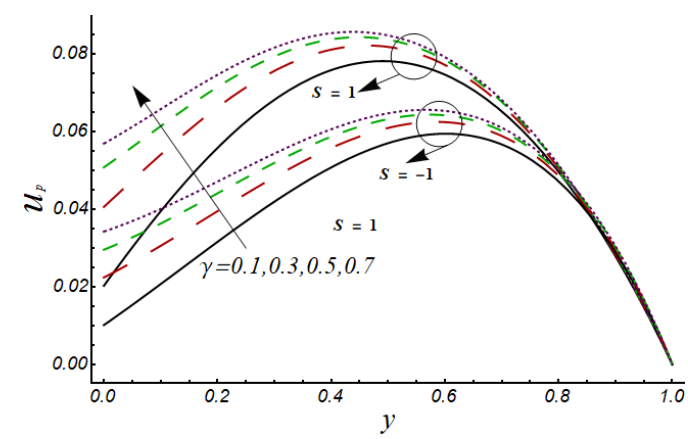

Fig. 3: (a) $u(y, t)$ (b) $u_{p}(y, t)$ for $\gamma$.

(a)

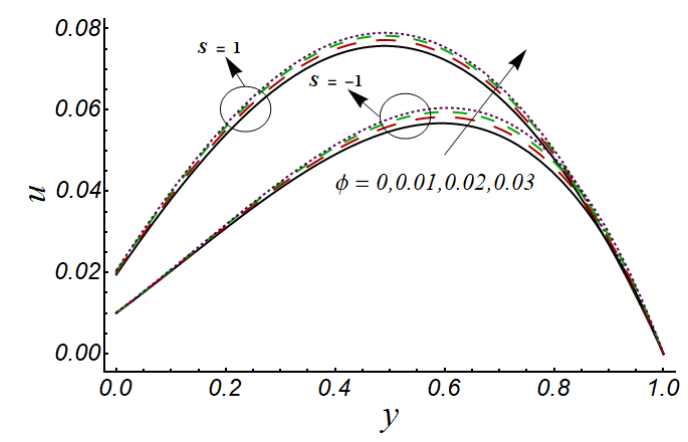

(b)

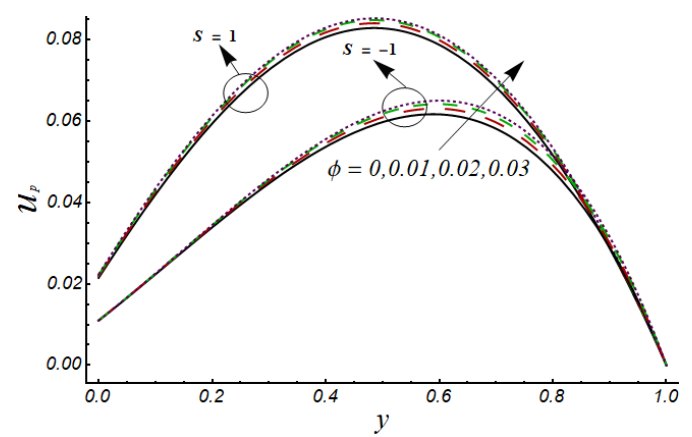

Fig. 4: (a) $u(y, t)$ (b) $u_{p}(y, t)$ for $\phi$.

(a)

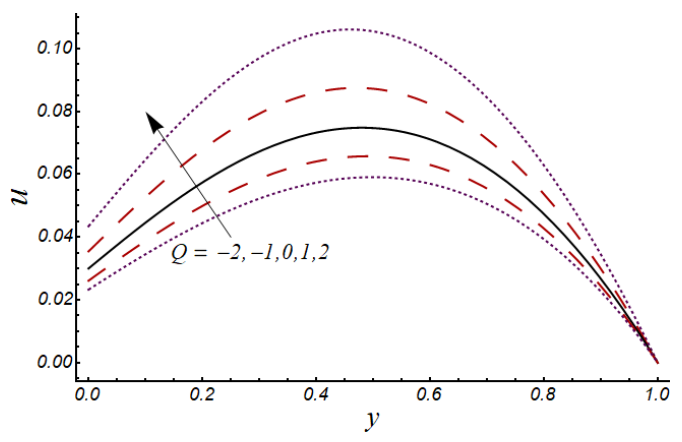

(b)

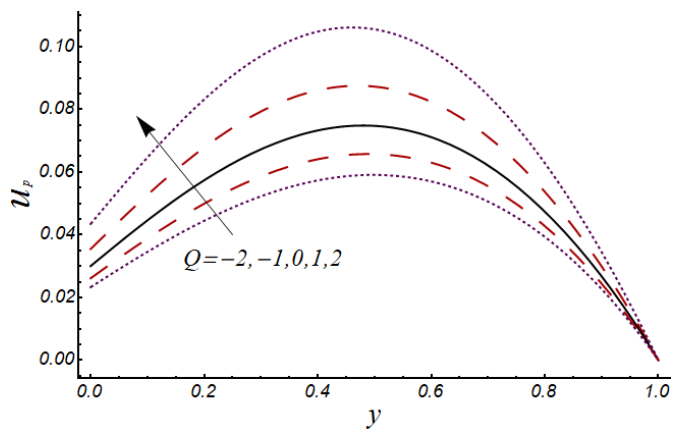

Fig. 5: (a) $u(y, t)$ (b) $u_{p}(y, t)$ for $Q$.

(a)

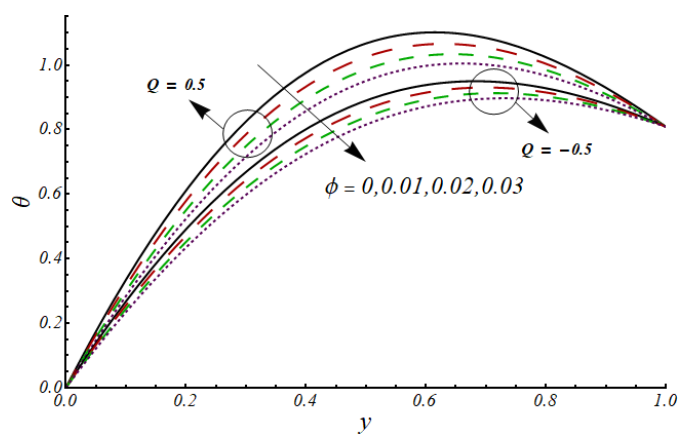

(b) 


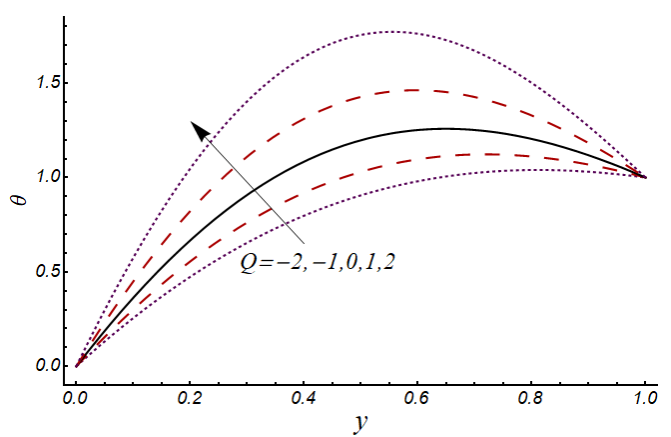

(c)

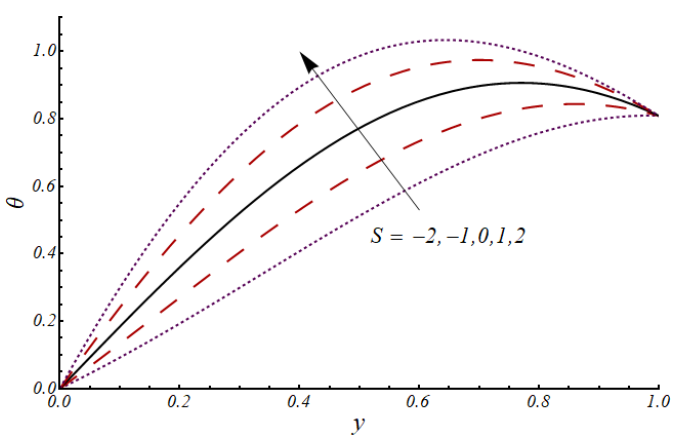

Fig. 6: $\theta(y, t)$ for (a) $\phi$ (b) $Q$ (c) $S$.

(a)

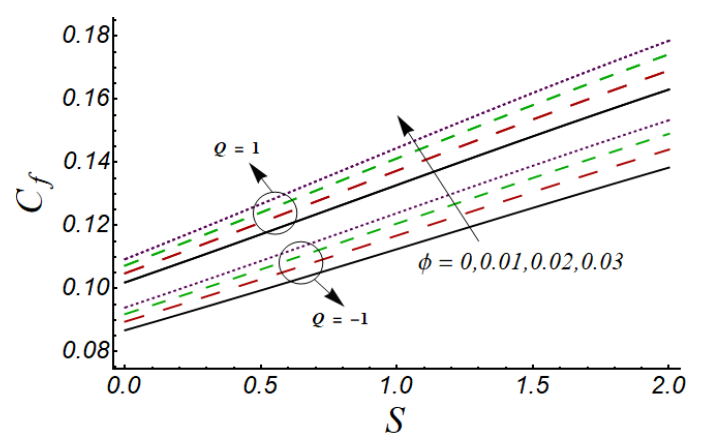

(b)

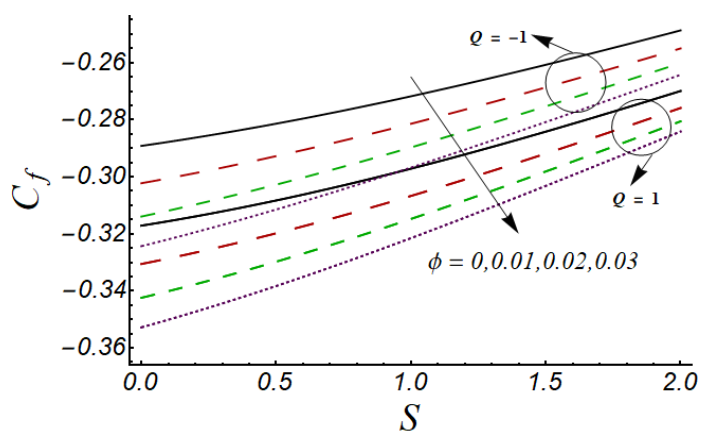

Fig. 7: $C_{f}$ for $\phi$ at (a) suction wall (b) injection wall.

(a)

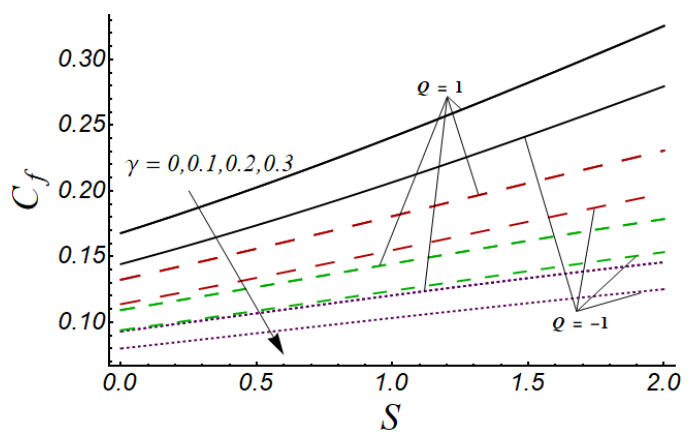

(b)

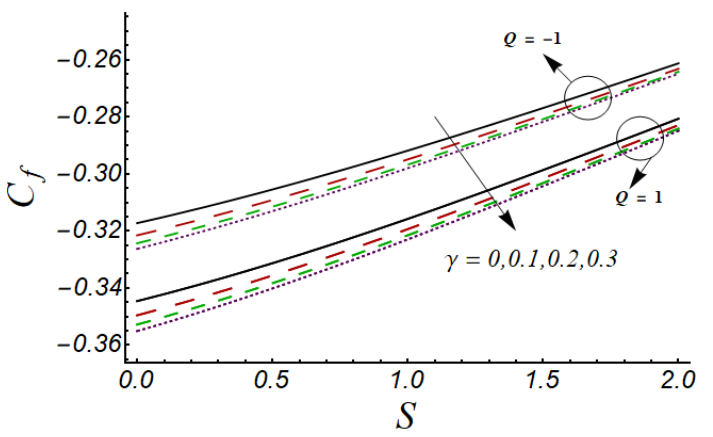

Fig. 8: $C_{f}$ for $\gamma$ at (a) suction wall (b) injection wall.

(a)

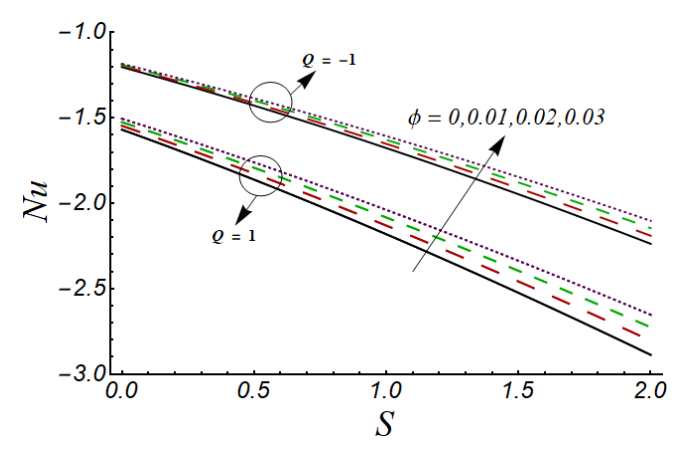

(b)

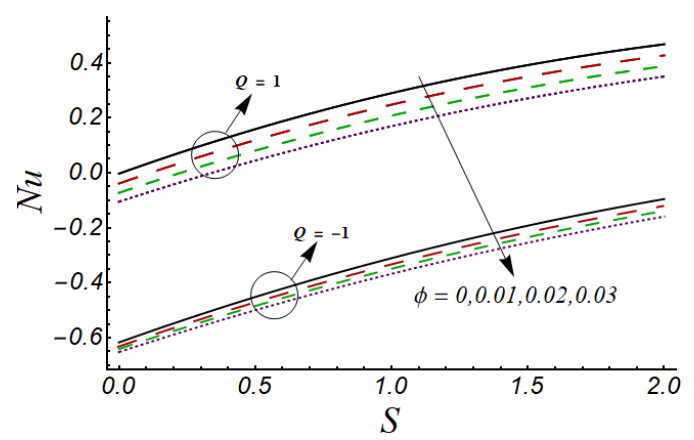

Fig. 9: $N u$ for $\phi$ at (a) suction wall (b) injection wall.

\section{Appendix}




$$
\begin{aligned}
& Q_{1}=1-\phi+\frac{\rho_{s} \phi}{\rho_{f}}, \quad Q_{2}=(1-\phi)^{2.5}, \quad Q_{3}=1-\phi+\frac{\phi \beta_{s}}{\beta_{f}}, \\
& Q_{4}=\frac{\sigma_{n f}}{\sigma_{f}}, \quad Q_{5}=1-\phi+\phi \frac{\left(\rho C_{p}\right)_{s}}{\left(\rho C_{p}\right)_{f}}, \quad Q_{6}=\frac{K_{n f}}{K_{f}}, \\
& Q_{7}=Q_{1} Q_{2}, \quad Q_{8}=\sqrt{\frac{\phi^{2}}{Q_{1} Q_{2}}+\frac{H^{2} Q_{4}}{Q_{1}}+\operatorname{Re} i \omega+\frac{L}{Q}\left(\frac{i \omega \operatorname{Re} M}{1+i \omega \operatorname{Re} M}\right)}, \\
& Q_{9}=\sqrt{N^{2}+Q-i \omega \operatorname{Pr} \operatorname{Re}}, \quad \mathrm{Q}_{10}=\operatorname{Pr} \rho Q_{5}, \\
& \mathrm{Q}_{11}=\frac{-Q-\sqrt{\mathrm{Q}_{10}{ }^{2}-4 Q_{6} Q_{9}{ }^{2}}}{2 Q_{6}}, \quad \mathrm{Q}_{12}=\frac{-Q+\sqrt{\mathrm{Q}_{10}{ }^{2}-4 Q_{6} Q_{9}{ }^{2}}}{2 Q_{6}}, \\
& C_{1}=\frac{1}{e^{Q_{11}}-e^{Q_{12}},} \quad C_{2}=-\frac{1}{e^{Q_{11}}-e^{Q_{12}}}, \\
& \mathrm{Q}_{13}=\frac{-Q_{7} S-\sqrt{4 Q_{8}{ }^{2} Q_{7}+Q_{7} S^{2}}}{2}, \\
& Q_{14}=\frac{-Q_{7} S+\sqrt{4 Q_{8}{ }^{2} Q_{7}+Q_{7} S^{2}}}{2}, \\
& Q_{15}=\frac{\lambda}{Q_{1} Q_{8}{ }^{2}}, \quad Q_{16}=\frac{-C_{1} G r Q_{3} Q_{7}}{Q_{11}{ }^{2}-Q_{7} Q_{8}^{2}+Q_{11} Q_{7} S}, \\
& Q_{17}=\frac{-C_{2} G r Q_{3} Q_{7}}{Q_{12}{ }^{2}-Q_{7} Q_{8}{ }^{2}+Q_{12} Q_{7} S}, \quad \mathrm{Q}_{18}=1-\gamma Q_{13}, \\
& \mathrm{Q}_{19}=1-\gamma Q_{14}, \quad \mathrm{Q}_{20}=Q_{15}+Q_{16}+Q_{17}-\gamma Q_{11} Q_{16}-\gamma Q_{12} Q_{17}, \\
& Q_{21}=Q_{15}+Q_{16} e^{Q_{11}}+Q_{17} e^{Q_{12}}, \\
& C_{3}=\frac{Q_{19} Q_{21}-e^{Q_{14}} Q_{20}}{e^{Q_{14}} Q_{18}-e^{Q_{13}} Q_{19}}, \quad C_{4}=\frac{e^{Q_{13}} Q_{20}-Q_{18} Q_{21}}{e^{Q_{14}} Q_{18}-e^{Q_{13}} Q_{19}} . \\
&
\end{aligned}
$$

\section{Concluding Remarks}

Analysis to examine the effects of slip on the oscillatory flow of a nanofluid in the presence of dusty particles is carried out. The radiative flux is also considered to explore the heat transmission characteristics of heatabsorbing/generating liquid. An exact approach is developed for the flow system. The numerical values are expressed in the form of graphs to demonstrate the impact of the associated physical parameters. The summary of the findings is as follows

- The velocities of fluid as well as dust particles upsurge with the enhanced values of slip and nanoparticle volume fraction parameters for both cases of injection and suction.

- The fluid and dust particles velocities grow for the case of suction and heat generation while they fall for the case of injection and heat absorption.

- Improved values of heat absorption, injection and nanoparticle volume fraction parameters reduces the fluid temperature while an opposite response is observed for suction and heat generation.

- The drag force at both channel walls enhances due to the nanoparticle volume fraction and suction.

- The improved value of slip lessens the surface friction at the suction wall of the channel.

- Heat transmission rate at the suction wall tends to increase with nanoparticle volume fraction while it reduces at the injection wall.

We are thankful to the reviewers for their encouraging comments and constructive suggestions to improve the quality of the manuscript. The authors appreciate the financial support from HEC Pakistan through SRGP-2109.

\section{References}

[1] M. R. Krishnamurthy, B. C. Prasannakumara, B. J. Gireesha, and R. S. R. Gorla, Effect of viscous dissipation on hydromagnetic fluid flow and heat transfer of nanofluid over an exponentially stretching sheet with fluid-particle suspension, Cogent Math., 2(1), 1050973, (2015) doi: 10.1080/23311835.2015.1050973

[2] S. Naramgari and C. Sulochana, MHD flow of dusty nanofluid over a stretching surface with volume fraction of dust particles, Ain Shams Eng. J. 7(2), 709-716, (2016) doi: 10.1016/j.asej.2015.05.015

[3] C. Sulochana and N. Sandeep, Flow and heat transfer behavior of MHD dusty nanofluid past a porous stretching/shrinking cylinder at different temperatures, J. Appl. Fluid Mech., 9(2), 543-553, (2016) doi: 10.18869/acadpub.jafm.68.225.24847

[4] B. C. Prasannakumara, N. S. Shashikumar, and P. Venkatesh, Boundary Layer Flow and Heat Transfer of fluid particle suspension with nanoparticles over a nonlinear stretching sheet embedded in a porous medium," Nonlinear Eng., 6(3), 179-190, (2017) doi: 10.1515/nleng-2017-0004

[5] B. J. Gireesha, B. Mahanthesh, and K. L. Krupalakshmi, Hall effect on two-phase radiated flow of magneto-dustynanoliquid with irregular heat generation /consumption Results Phys., 7, 4340-4348 (2017) doi: 10.1016/j.rinp.2017.08.040

[6] Z. A. S. Raizah, Natural convection of dusty hybrid nanofluids in an enclosure including two oriented heated fins," Appl. Sci., 9(13) (2019) doi: 10.3390/app9132673

[7] H. Kaneez, J. Alebraheem, A. Elmoasry, R. S. Saif, and M. Nawaz, Numerical investigation on transport of momenta and energy in micropolar fluid suspended with dusty, mono and hybrid nano-structures, AIP Adv., 10(4) 4, 2020, doi: 10.1063/5.0003042.

[8] M. Radhika, R. J. Punith Gowda, R. Naveenkumar, Siddabasappa, and B. C. Prasannakumara, Heat transfer in dusty fluid with suspended hybrid nanoparticles over a melting surface, Heat Transf., 50, 2159-2167 (2021) doi: $10.1002 / \mathrm{htj} .21972$

[9] T. Hayat, R. Naz, and A. Alsaedi, Effects of slip condition in the channel flow of nanofluid, $J$. Comput. Theor. Nanosci., 11(12) 2618-2624 (2014) doi: 
$10.1166 /$ jctn.2014.3686

[10] M. H. Kamel, I. M. Eldesoky, B. M. Maher, and R. M. Abumandour, Slip Effects on Peristaltic Transport of a Particle-Fluid Suspension in a Planar Channel, Appl. Bionics Biomech., $2015 \quad$ (2015) doi: 10.1155/2015/703574

[11] M. Guria, Effect of slip condition on vertical channel flow in the presence of radiation, Int. J. Appl. Mech. Eng., 21(2) 341-358 (2016) doi: 10.1515/ijame-20160021

[12]P. Panaseti and G. C. Georgiou, Viscoplastic flow development in a channel with slip along one wall, J. non-Newton. Fluid Mech., 248 8-22 (2017) doi: 10.1016/j.jnnfm.2017.08.008

[13] K. Pravin Kashyap, O. Ojjela, and S. K. Das, MHD slip flow of chemically reacting UCM fluid through a dilating channel with heat source/sink, Nonlinear Eng., 8(1) 523 533 (2019) doi: 10.1515/nleng-2018-0036

[14] N. Saleem, S. Akram, F. Afzal, E. H. Aly, and A. Hussain, Impact of velocity second slip and inclined magnetic field on peristaltic flow coating with jeffrey fluid in tapered channel, Coatings, 10(1) (2020) doi: 10.3390/coatings 10010030

[15] M. Y. Malik, M. Bibi, F. Khan, and T. Salahuddin, Numerical solution of Williamson fluid flow past a stretching cylinder and heat transfer with variable thermal conductivity and heat generation/absorption, AIP Adv., 6(3) (2016) doi: 10.1063/1.4943398

[16] A. K. Pandey and M. Kumar, MHD flow inside a stretching/shrinking convergent/divergent channel with heat generation/absorption and viscous-ohmic dissipation utilizing CU-water nanofluid, Comput. Therm. Sci., (10)5 457-471 (2018) doi: 10.1615/ComputThermalScien. 2018020807

[17] B. K. Jha and P. B. Malgwi, Couette flow and heat transfer of heat-generating / absorbing fluid in a rotating channel in presence of viscous dissipation, Arab J. Basic Appl. Sci., 27(1) 67-74 (2020) doi: 10.1080/25765299.2020.1715147

[18] A. Mishra, A. K. Pandey, A. J. Chamkha, and M. Kumar, Roles of nanoparticles and heat generation/absorption on $\mathrm{MHD}$ flow of $\mathrm{Ag}-\mathrm{H} 2 \mathrm{O}$ nanofluid via porous stretching/shrinking convergent/divergent channel, J. Egypt. Math. Soc., 28(1) (2020), doi: 10.1186/s42787020-00079-3

[19] D. Prakash, N. Elango, and I. S. Hussain, Effect of heat generation on MHD free convective flow of viscous fluid in a vertical channel in the presence of variable properties, in AIP Conference Proceedings, 2277 (2020) doi: $10.1063 / 5.0025228$

[20]A. C. Cogley, W. G. Vincenti, and S. E. Gilles, Differential approximation for radiative transfer in a nongrey gas near equilibrium, AIAA J., 6(3) 551-553 (1968) doi: 10.2514/3.4538.

[21] A. Roja and B. J. Gireesha, Impact of Hall and Ion effects on MHD couple stress nanofluid flow through an inclined channel subjected to convective, hydraulic slip, heat generation, and thermal radiation, Heat Transf., 49 3314-3333 (2020) doi: 10.1002/htj.21775 